\title{
Efficacy of Miniuniportal Video-Assisted Thoracoscopic Selective Sympathectomy (Ramicotomy) for the Treatment of Severe Palmar and Axillar Hyperhidrosis
}

\author{
Ali Akil ${ }^{1} \quad$ Michael Semik $^{1} \quad$ Stefan Fischer ${ }^{1}$ \\ ${ }^{1}$ Department of Thoracic Surgery and Lung Support, Ibbenbueren \\ General Hospital, Ibbenbueren, Germany \\ Thorac Cardiovasc Surg 2019;67:415-419.
}

\begin{abstract}
Address for correspondence Ali Akil, MD, Department of Thoracic Surgery and Lung Support, Klinikum Ibbenbüren, Grosse Strasse 41, 49477 Ibbenbueren, Germany (e-mail: dr.ali.akil.11@gmail.com).
\end{abstract}

\begin{abstract}
Background Video-assisted thoracoscopic surgery (VATS) clipping of the sympathetic branch has become the standard approach for the treatment of essential hyperhidrosis when conservative treatment failed. However, this is associated with relevant potential complications such as postoperative compensatory sweating and recurrent sweating. We report the outcome after selective sympathectomy (ramicotomy) through a miniuniportal VATS approach in patients with therapy-refractory palmar and/or axillary hyperhidrosis.

Methods A total of 51 consecutive patients ( 37 females, mean age: 30 years, range: 12-64 years) who suffered from therapy-refractory palmar and/or axillary severe hyperhidrosis were included. Data were prospectively collected and retrospectively analyzed. All patients underwent bilateral miniuniportal VATS ramicotomy. Duration of surgery, hospital stay, recurrent, and compensatory sweating were documented.

Results All patients had palmar sweating, where $51 \%$ had additional axillary sweating and $57 \%$ had additional plantar sweating. In all patients, selective division of the rami communicantes of the thoracic sympathetic ganglions Th2 to Th5 was performed. The mean duration of bilateral surgery for both sides was $67 \pm 2.5$ minutes. The mean

Keywords

- hyperhidrosis

- video-assisted thoracoscopic surgery

- sympathetic nerve

- ramicotomy postoperative hospital stay was $2 \pm 1$ days. After surgery and at further follow-up (mean: $12 \pm 2.5$ months), all patients presented dry and warm hands and axillae, without any evidence of compensatory or recurrent sweating. All patients described a remarkable increase in quality of life.

Conclusion Miniuniportal VATS ramicotomy represents a feasible surgical technique with a very high success and satisfaction rate. Therefore, this approach should be considered as the method of choice for the treatment of patients with severe therapyrefractory palmar and axillary hyperhidrosis.
\end{abstract}

\section{Introduction}

Severe primary hyperhidrosis is commonly associated with excessive sweating beyond physiological needs, thereby especially affecting the palmar, plantar, and axillary regions. $^{1}$

received

November 25, 2017

accepted after revision

February 22, 2018

published online

May 8, 2018
It has been clearly shown that it influences many aspects of patient's life. The psychosocial impact leading to difficulties in social life, at work, at school, and through daily activities is significant. ${ }^{2}$ Surgery of the sympathicus chain was first introduced in the 19th century for the treatment of an epileptic patient. ${ }^{3}$ However, the first sympathectomy for

(c) 2019 Georg Thieme Verlag KG Stuttgart · New York
DOI https://doi.org/ 10.1055/s-0038-1642030. ISSN 0171-6425. 
hyperhidrosis was performed in $1920 .{ }^{4}$ With the development of minimally invasive procedures, Hughes reported the first thoracoscopic sympathectomy in $1942 .{ }^{5}$ Since then, the surgery of the sympathetic nerve has developed progressively and the knowledge on the physiology on the sympathetic nerve has evolved. Several surgical approaches have been developed to treat patients with severe palmar, axillary, and plantar hyperhidrosis. However, it remains unclear which the ideal procedure is, especially to avoid the undesirable postoperative complication of compensatory or recurrent sweating. ${ }^{6-9}$ Here, we report our initial experience using selective sympathetic ramicotomy for patients suffering from severe therapy-refractory palmar and axillary primary hyperhidrosis, which was performed through a novel miniuniportal video-assisted thoracoscopic surgery (VATS) approach. Furthermore, we demonstrate the longterm efficacy.

\section{Patients and Methods}

Between January 2014 and October 2017, a total of 51 patients ( $n=37$ females) with severe essential palmar and/or axillary hyperhidrosis were prospectively enrolled in this study and data were recorded. The mean age was 30 years (range: $12-64$ years). All patients were consented to the study preoperatively. Exclusion criteria were defined as: age $<15$ years, pregnancy and excessive sweating due to a specific health issue such as late onset of sweating combined with the presence of comorbidities or the use of medication known to be associated with sweating.

Prior to surgery, all patients underwent various nonsurgical therapies such as topical application of aluminum chloride, iontophoresis, oral anticholinergics, and botulinum toxin injection, which all failed or lead to recurrent sweating within 3 months. Primary end point of this study was the postoperative absence of palmar and axillary sweating at 4 weeks after the surgical procedure. Secondary end points included the duration of surgery, hospital stay, length of chest tube therapy, and compensatory sweating. All patients were postoperatively evaluated 4 weeks after surgery. Further follow-up was performed by telephone questionnaire.

\section{Surgical Technique}

Bilateral miniuniportal VATS ramicotomy ( - Video 1) was performed through a $1-\mathrm{cm}$ incision at the middle of the transverse axillary line via the third intercostal space (-Fig. 1). A 5-mm trocar was inserted into the thoracic cavity to guide the $5 \mathrm{~mm}$ thoracoscope. - Fig. 2 presents the sympathetic chain with the rami communicantes of the sympathetic ganglions. Through the same incision, a 5-mm endoscopic, monopolar, electrocautery hook was inserted. After visual identification of the sympathetic chain, the parietal pleura was incised with 5 to $10 \mathrm{~mm}$ distance from the main sympathetic chain. Further on, the rami communicantes (Th2-Th5) were transected with electric cautery in $5 \mathrm{~mm}$ distance from the sympathetic ganglion. Thereby, the main sympathetic trunk remained intact. The intercostal space and its neurovascular structures were

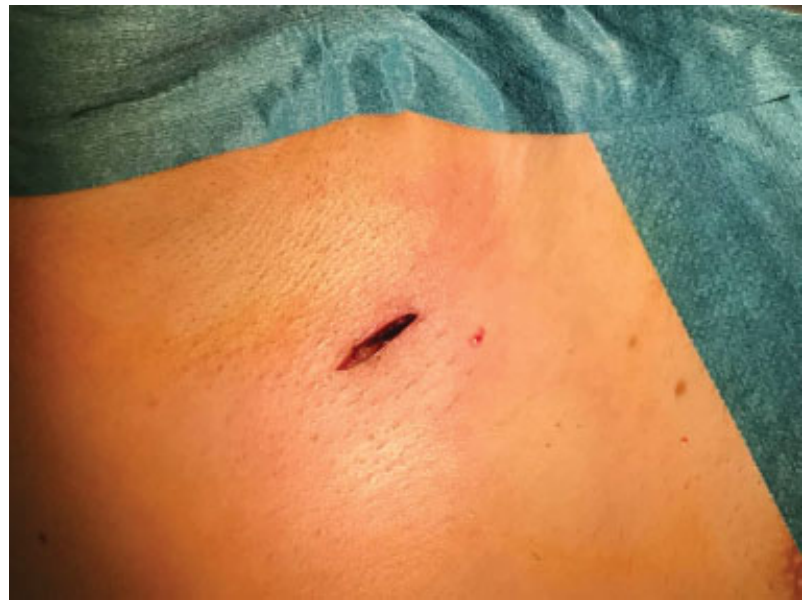

Fig. 1 Video-assisted thoracoscopic surgery through a 1-cm incision for inserting a 5-mm trocar and 5-mm electrocautery device.

also preserved (-Fig. $\mathbf{3}$ ). - Fig. 4 shows the preserved sympathetic chain at the end of the ramicotomy.

\section{Video 1}

Video session demonstrating intraoperative steps for thoracoscopic selective ramicotomy: Using single lung ventilation of the opposite lung, the thoracic sympathetic chain is visualized. Additionally, in support of lung deflation and to achieve an excellent view, the lung is gently pushed forward using a thoracoscopic sponge stick. The sympathetic chain with the rami communicantes is now identified from the second to the fifth rib after identification of the levels using a hook to palpate the ribs. After that, the parietal pleura overlying the main sympathetic chain is incised from Th2 to Th5 using an endoscopic, electric cautery hook. With the sponge stick, the main sympathetic chain is gently pulled down. After visual identification, the rami communicantes will be transected from Th2 to Th5 using electric cautery. At the end of the ramicotomy, the main sympathetic chain and the neurovascular structured are preserved. For pain assessment, carbostesin $0.25 \%$ is injected into the intercostal space. Online content including video sequences viewable at: https://www.thieme-connect.com/products/ejournals/ html/doi/Online content including video sequences viewable at: https://www.thieme-connect.com/ products/ejournals/html/doi/10-1055-s-0038-1642030tcs-17-5014-v1.mp4.

\section{Results}

Preoperative, severe palmar sweating was presented in 51 patients; 26 patients suffered simultaneously from severe palmar and axillary sweating. From the 51 patients, 28 patients presented additionally with severe or moderate 


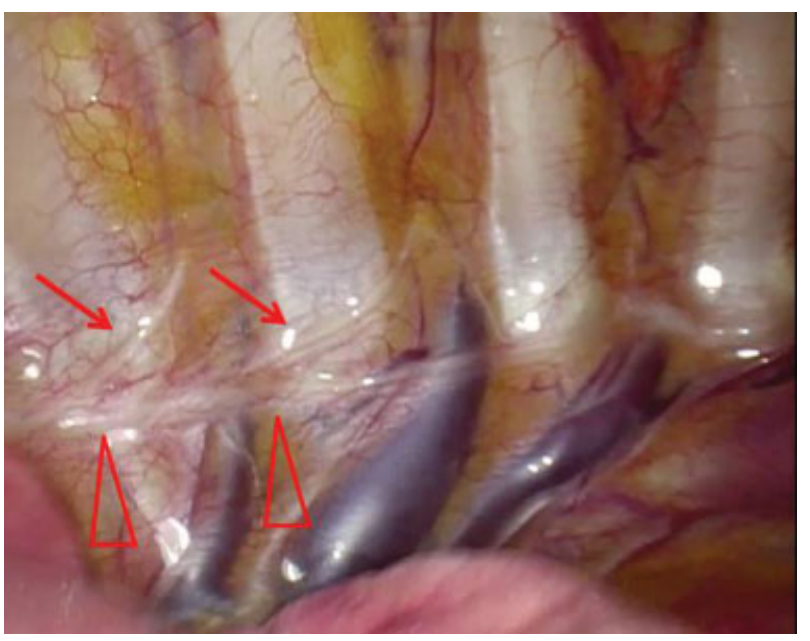

Fig. 2 The sympathetic chain (red triangle) and the rami communicantes (red arrows) as well as the second till fifth rip. This serves as anatomic landmarks for defining the area of resection.

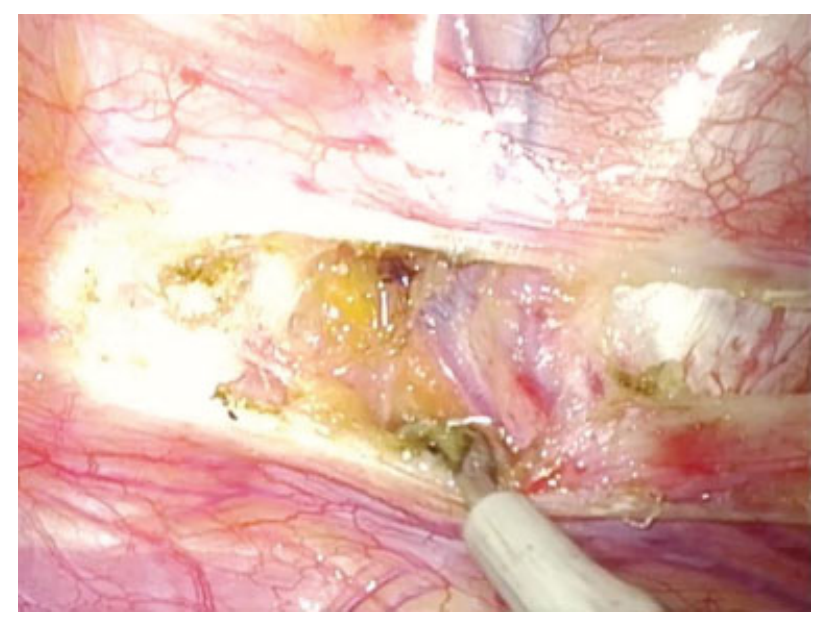

Fig. 3 The intercostal space and its neurovascular structures, which were also preserved after ramicotomy.

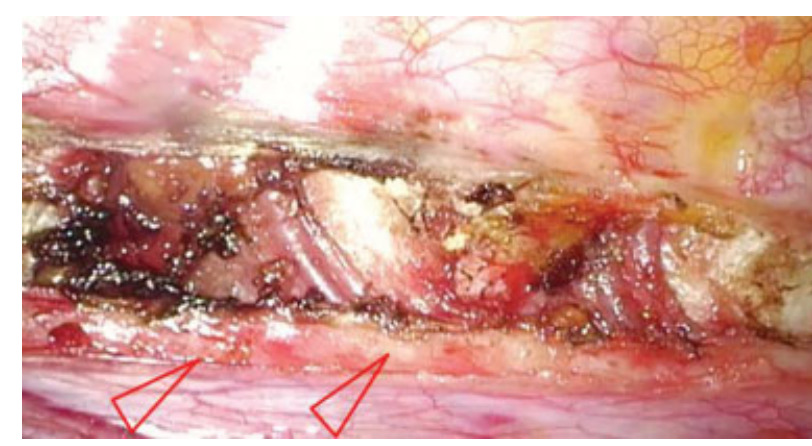

Fig. 4 Preserved sympathetic chain after resection of the rami communicantes from the second ganglion to the fifth ganglion.
Table 1 Location of sweating

\begin{tabular}{|l|l|l|}
\hline Location of sweating & $\%$ & Number of patients \\
\hline Palmar & 100 & 51 \\
\hline Palmar and axillary & 51 & 26 \\
\hline Palmar and plantar & 55 & 28 \\
\hline Palmar, axillary, and plantar & 35 & 18 \\
\hline
\end{tabular}

plantar sweating. The location of hyperhidrosis is summarized in - Table 1. In all patients, the intra- and postoperative courses were uneventful. One patient required chest tube drainage for 5 days due to prolonged air leak. The mean duration of bilateral surgery was $67 \pm 2.5$ minutes. The mean hospital stay was $2 \pm 1$ days. Chest tubes were removed after no air leakage was detected by the digital system with a postoperative mean duration of 1 day (range: 0-5 days). After chest tube removal, chest $\mathrm{X}$-ray did not show any evidence for pneumothorax or pleural effusion in all patients. After the procedure, all patients experienced total palmar and axillary dryness. Interestingly, in 10 female patients, plantar sweating diminished completely, which was not expected. At the time of discharge, all patients reported warm and dry hands and axillae. This was identical with findings at 4 weeks after surgery, where no evidence of recurrent palmar or axillary sweating was seen. Furthermore, none of the patients reported evidence of compensatory sweating. Moreover, further follow-up did not show any palmar and axillary sweating or compensatory sweating. The mean follow-up was $12 \pm 2.5$ months after surgery.

\section{Discussion}

Primary hyperhidrosis is a pathological state of excessive sweating potentially affecting different regions especially the hands, the axilla, and the feet. Genetic predisposition was discussed. ${ }^{10} \mathrm{~A}$ variety of nonsurgical treatment options such as the application of anticholinergic drugs, botulinum toxin A injection, iontophoresis, aluminum-based antiperspirants, and laser therapy were described for treating primary hyperhidrosis and are routinely applied initially. ${ }^{11}$ However, in a certain number of patients, these conservative approaches fail or lead to early recurrence. In this study, all patients underwent intensive conservative therapy managed by a dermatologist for several months, but did not show any remarkable improvement of their sweating. Patients were then introduced to the option of surgical therapy.

Surgical techniques for primary hyperhidrosis regarding the sympathetic nerve have developed continuously during the past decades. Techniques to interrupt the sympathetic chain are well described and vary from sympathectomy, "sympathicotomy" or "sympathotomy," sympathetic block to ramicotomy. The surgical term of "sympathectomy" presents the dissection of a specific part of the sympathetic trunk and ganglia. It was widely spread and a standard during the initial open surgical approaches. Today, the most frequently used technique is the "sympathicotomy" or "sympathotomy," which is based on the interruption of the sympathetic trunk only by 
its complete transection, using electrocautery, endoscopic scissors, or other devices. ${ }^{12-14}$ Furthermore, the sympathetic block is performed using metallic clips, which are applied across the sympathetic nerve without transection. ${ }^{6}$ However, the most effective and least invasive surgical approach is not clearly defined. An alternative is the ramicotomy, where the sympathetic rami communicantes of the sympathetic ganglions are selectively resected, while preserving the thoracic sympathetic ganglia and the nerve chain. ${ }^{8}$ This has been reported to reduce compensatory sweating. However, some studies report a higher recurrence rate. ${ }^{15}$

With regard to the anatomic level of nerval interruption of the sympathetic chain, several studies suggested multiple levels. It has been reported that spinal segments 3 to 6 seem to be the origin of the preganglionic fibers reaching the arm and hand. Especially, the third and fourth segments are the most dominant origins. ${ }^{16}$ Yazbek et al compared the effects of sympathectomy at T2 and T3, addressing that sparing the T2 ganglion might decrease the risk of compensatory sweating. ${ }^{17}$ Other studies reported that isolating the T3 and T4 ganglions may lead to acceptable results in the treatment of palmar hyperhidrosis. However, the rate of compensatory sweating in these studies varies. ${ }^{18-20}$ Therefore, the Society of Thoracic Surgeons has published a consensus paper for the surgical treatment of hyperhidrosis based on a broad review of the literature. The report suggested a standardized surgical approach for primary hyperhidrosis. Moreover, the exact sympathetic nerve levels for interruption according to the corresponding sweating regions were defined. ${ }^{21}$ Interruption at R4 to R5 levels is supposed to be the most effective in patients suffering from palmar and plantar hyperhidrosis. Patients with isolated axillary hyperhidrosis should be exposed to nonsurgical treatment options before they undergo surgical procedures. However, if treatment fails and patients are subjected to surgery, R4 to R5 interruption is recommended. Furthermore, for facial blushing/hyperhidrosis, R2 to R3 interruption is suggested to be the most effective surgical approach but with increased risk for compensatory sweating.

Regardless of the previously reported experience and therapy strategies, in our study, the rami communicantes of the sympathetic ganglions were interrupted at the $\mathrm{R} 2$ to R5 levels independently of the region of sweating. Therefore, patients with palmar or axillary hyperhidrosis underwent the same surgical approach. However, patients with only plantar sweating were not operated. The primary focus of the surgical approach in this study was to treat predominantly palmar and/or axillary hyperhidrosis.

Gorur et al compared the outcome between patients who underwent T3/T4 sympathetic clipping with those undergoing only T3 and T6 clipping. Both groups were compared regarding compensatory sweating and recovery of plantar hyperhidrosis. Notably, compensatory sweating rate was high in both groups, but recovery of plantar hyperhidrosis was higher in patients with $\mathrm{T} 3$ and $\mathrm{T} 6$ clipping. This analysis revealed that clipping beyond the thoracic level T6 could be effective in reducing plantar sweating. ${ }^{22}$ Recently, Xiao et al evaluated the effect of VATS sympathectomy at the thoracic level T4 on plantar hyperhidrosis for patients with palmo- plantar hyperhidrosis with follow-up after 1 and 6 months. Improvements in plantar hyperhidrosis were observed initially in singular patients ( $28 \%, 8$ out of 28 patients), but this improvement has not sustained over a long period. ${ }^{23}$ Till now improvement of plantar hyperhidrosis after selective ramicotomy of T2 till T5 ganglion has not been reported. In our study, we observed an improvement in plantar hyperhidrosis ( $n=10$ patients), without sweating recurrence during the follow-up period. This pathophysiological finding, however, cannot be explained by the current scientific knowledge.

Although the interrupted levels were extensive in comparison to other studies, compensatory sweating or even excessive sweating in other body regions were not observed. Even in further follow-up, no recurrent or compensatory sweating was observed. Even though we picked R2 to R5 for interruption on the level of rami communicantes, we fully preserved the sympathetic chain as a difference.

Although a ramicotomy seems to be a well-tolerated surgical approach without any side effects such as compensatory sweating and recurrent sweating, we address the following limitations of this study. The number of patients recruited is limited and represents our initial experience. Further studies on larger cohorts are needed to verify our promising results. Also, a control group was not added, which is essential for further investigations. Contrary to previous reports, we have achieved promising postoperative results. Taking the limitation of this study into account, we now offer this unique uniportal VATS approach to patients with severe palmar, axillary, and otherwise therapy-refractory hyperhidrosis.

In conclusion, this surgical approach is safe, cosmetically most favorable and leads to instant patient satisfaction and increase in quality of life in a cohort of individuals that have gone through long-term treatments and are often accompanied by psychosocial conflicts and depression.

\section{Conflict of Interest}

None to be declared.

\section{References}

1 Baumgartner FJ, Toh Y. Severe hyperhidrosis: clinical features and current thoracoscopic surgical management. Ann Thorac Surg 2003;76(06):1878-1883

2 Kamudoni P, Mueller B, Halford J, Schouveller A, Stacey B, Salek MS. The impact of hyperhidrosis on patients' daily life and quality of life: a qualitative investigation. Health Qual Life Outcomes 2017;15(01):121

3 Alexander W. The Treatment of Epilepsy. Edinburgh: Young J. Pentland; 1889:27-106

4 Seguin A, Martinod E, Azorin JF. Sympathectomic thoracique par videothoracoscopic del'hyperhidrose. Sang Thrombox Vaisseaux 2003;15(04):187-192

5 Hughes J. Endothoracic sympathectomy. Proc R Soc Med 1942; 35(09):585-586

6 Lin CC, Mo LR, Lee LS, Ng SM, Hwang MH. Thoracoscopic T2sympathetic block by clipping-a better and reversible operation for treatment of hyperhidrosis palmaris: experience with 326 cases. Eur J Surg Suppl 1998;580(580):13-16

7 Coelho MdeS, Silva RF, Mezzalira G, et al. T3T4 endoscopic sympathetic blockade versus T3T4 video thoracoscopic sympathectomy in the treatment of axillary hyperhidrosis. Ann Thorac Surg 2009; 88(06):1780-1785 
8 Cho HM, Chung KY, Kim DJ, Lee KJ, Kim KD. The comparison of VATS ramicotomy and VATS sympathicotomy for treating essential hyperhidrosis. Yonsei Med J 2003;44(06):1008-1013

9 Bryant AS, Cerfolio RJ. Satisfaction and compensatory hyperhidrosis rates 5 years and longer after video-assisted thoracoscopic sympathotomy for hyperhidrosis. J Thorac Cardiovasc Surg 2014; 147(04):1160-1163.e1

10 Ro KM, Cantor RM, Lange KL, Ahn SS. Palmar hyperhidrosis: evidence of genetic transmission. J Vasc Surg 2002;35(02): 382-386

11 Kurta AO, Glaser DA. Emerging nonsurgical treatments for hyperhidrosis. Thorac Surg Clin 2016;26(04):395-402

12 de Campos JR, Wolosker N, Yazbek G, et al. Comparison of pain severity following video-assisted thoracoscopic sympathectomy: electric versus harmonic scalpels. Interact Cardiovasc Thorac Surg 2010;10(06):919-922

13 Fiorelli A, D'Aponte A, Canonico R, et al. T2-T3 sympathectomy versus sympathicotomy for essential palmar hyperhidrosis: comparison of effects on cardio-respiratory function. Eur J Cardiothorac Surg 2012;42(03):454-461

14 Byrne J, Walsh TN, Hederman WP. Endoscopic transthoracic electrocautery of the sympathetic chain for palmar and axillary hyperhidrosis. Br J Surg 1990;77(09):1046-1049

15 Gossot D, Toledo L, Fritsch S, Célérier M. Thoracoscopic sympathectomy for upper limb hyperhidrosis: looking for the right operation. Ann Thorac Surg 1997;64(04):975-978
16 Gray H. The sympathetic nerves. Anatomy of the Human Body. Philadelphia: Lea \& Febiger; 2000:1292-1299

17 Yazbek G, Wolosker N, de Campos JR, Kauffman P, Ishy A, PuechLeão P. Palmar hyperhidrosis-which is the best level of denervation using video-assisted thoracoscopic sympathectomy: T2 or T3 ganglion? J Vasc Surg 2005;42(02):281-285

18 Lee DY, Kim DH, Paik HC. Selective division of T3 rami communicantes (T3 ramicotomy) in the treatment of palmar hyperhidrosis. Ann Thorac Surg 2004;78(03):1052-1055

19 Ishy A, de Campos JR, Wolosker N, et al. Objective evaluation of patients with palmar hyperhidrosis submitted to two levels of sympathectomy: T3 and T4. Interact Cardiovasc Thorac Surg 2011;12(04):545-548

20 Liu Y, Yang J, Liu J, et al. Surgical treatment of primary palmar hyperhidrosis: a prospective randomized study comparing $\mathrm{T} 3$ and T4 sympathicotomy. Eur J Cardiothorac Surg 2009;35(03):398-402

21 Cerfolio RJ, De Campos JR, Bryant AS, et al. The Society of Thoracic Surgeons expert consensus for the surgical treatment of hyperhidrosis. Ann Thorac Surg 2011;91(05):1642-1648

22 Gorur R, Yiyit N, Yildizhan A, et al. Is T3 and T6 sympathetic clipping more effective in primary palmoplantar hyperhydrosis? Thorac Cardiovasc Surg 2011;59(06):357-359

23 Xiao P, Liu A, Liu W. Effect of T4 thoracoscopic sympathectomy on plantar hyperhidrosis in patients with primary palmoplantar hyperhidrosis [in Chinese]. Zhong Nan Da Xue Xue Bao Yi Xue Ban 2016;41(03):300-304 